OPEN ACCESS

Approved by: Frontiers Editorial Office,

Frontiers Media SA, Switzerland

*Correspondence:

Jianhua Lin

Jianhual@126.com

Specialty section:

This article was submitted to

Pharmacology of Anti-Cancer Drugs,

a section of the journal

Frontiers in Pharmacology

Received: 04 August 2021

Accepted: 18 August 2021

Published: 02 September 2021

Citation:

Huang $Z$, Chen $H$, Wang S, Wei H,

Wang $X$, Shen $R$, Wang $Y$, Lin $R$ and

Lin J (2021) Corrigendum: NLRP3 Overexpression Associated With Poor

Prognosis and Presented as an

Effective Therapeutic Target

in Osteosarcoma.

Front. Pharmacol. 12:753231. doi: 10.3389/fphar.2021.753231

\section{Corrigendum: NLRP3 Overexpression Associated With Poor Prognosis and Presented as an Effective Therapeutic Target in Osteosarcoma}

\begin{abstract}
Zhen Huang ${ }^{1,2,3}$, Hui Chen ${ }^{4}$, Shenglin Wang ${ }^{3}$, Hongxiang Wei ${ }^{3}$, Xinwen Wang ${ }^{5}$, Rongkai Shen ${ }^{3}$, Yunqing Wang ${ }^{3}$, Rongjin Lin $^{6}$ and Jianhua Lin ${ }^{2,3 *}$

${ }^{1}$ Department of Rehabilitation, The First Affiliated Hospital of Fujian Medical University, Fuzhou, China, ${ }^{2}$ Department of Orthopedics, The First Affiliated Hospital of Fujian Medical University, Fuzhou, China, ${ }^{3}$ Fujian Orthopedics Research Institution, The First Affiliated Hospital of Fujian Medical University, Fuzhou, China, ${ }^{4}$ Department of Nephrology, Shanghai East Hospital, School of Medicine, Tongji University, Shanghai, China, ${ }^{5}$ Department of Orthopedics, The People's Hospital of Jiangmen City, Southern Medical University, Jiangmen, China, ${ }^{6}$ Department of Nursing, The First Affiliated Hospital of Fujian Medical University, Fuzhou, China
\end{abstract}

Keywords: osteosarcoma, prognosis, NLRP3, CY-09, lentivirus

\section{A Corrigendum on}

NLRP3 Overexpression Associated With Poor Prognosis and Presented as an Effective Therapeutic Target in Osteosarcoma

by Huang, Z., Chen, H., Wang, S., Wei, H., Wang, X., Shen, R., Wang, Y., Lin, R., and Lin, J. (2021). Front. Pharmacol. 12:724923. doi: 10.3389/fphar.2021.724923

In the original article, there was a mistake in Figure 3 as published. In the process of rearranging our published articles, we found a problem by accident that the tubulin blots in Figure 3B were mistakenly uploaded. The corrected Figure 3 appears below.

The authors apologize for this error and state that this does not change the scientific conclusions of the article in any way. The original article has been updated.

Publisher's Note: All claims expressed in this article are solely those of the authors and do not necessarily represent those of their affiliated organizations, or those of the publisher, the editors and the reviewers. Any product that may be evaluated in this article, or claim that may be made by its manufacturer, is not guaranteed or endorsed by the publisher.

Copyright $\odot 2021$ Huang, Chen, Wang, Wei, Wang, Shen, Wang, Lin and Lin. This is an open-access article distributed under the terms of the Creative Commons Attribution License (CC BY). The use, distribution or reproduction in other forums is permitted, provided the original author(s) and the copyright owner(s) are credited and that the original publication in this journal is cited, in accordance with accepted academic practice. No use, distribution or reproduction is permitted which does not comply with these terms. 


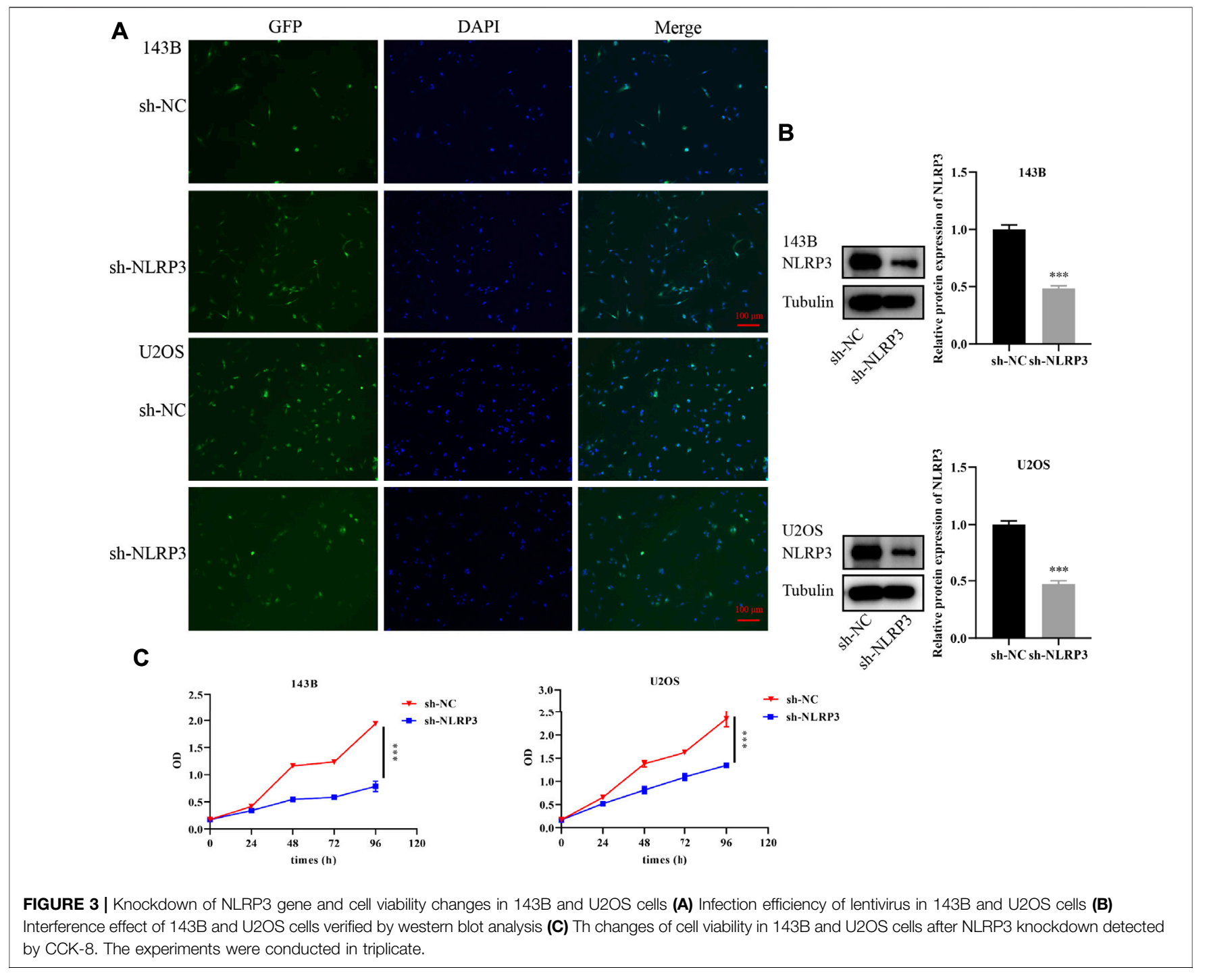

